PROCEEDINGS OF THE

AMERICAN MATHEMATICAL SOCIETY

Volume 129, Number 8, Pages 2451-2460

S 0002-9939(00)05822-6

Article electronically published on December 7, 2000

\title{
HYPOELLIPTIC RANDOM HEAT KERNELS: A CASE STUDY
}

\author{
RICHARD B. SOWERS
}

(Communicated by Claudia Neuhauser)

\begin{abstract}
We consider the fundamental solution of a simple hypoelliptic stochastic partial differential equation in which the first-order term is modulated by white noise. We derive some short-time asymptotic formulæ. We discover that the form of the dominant short-time asymptotics depends nontrivially upon the interplay between the geometry of the noisy first-order term and the geometry defined by the hypoelliptic operator.
\end{abstract}

\section{INTRODUCTION}

Consider $\mathbb{R}^{2}$ with its standard Euclidean atlas. Define two vector fields

Note that

$$
\begin{aligned}
& A_{1}(x, y) \stackrel{\text { def }}{=} \frac{\partial}{\partial x}, \\
& A_{2}(x, y) \stackrel{\text { def }}{=} x \frac{\partial}{\partial y}, \quad(x, y) \in \mathbb{R}^{2} .
\end{aligned}
$$

so that

$$
A_{3}(x, y) \stackrel{\text { def }}{=}\left[A_{1}, A_{2}\right](x, y)=\frac{\partial}{\partial y}, \quad(x, y) \in \mathbb{R}^{2},
$$

$$
\operatorname{Span}\left\{\operatorname{Lie}\left\{A_{1}, A_{2}\right\}\right\}(x, y)=T_{(x, y)} \mathbb{R}^{2}, \quad(x, y) \in \mathbb{R}^{2} .
$$

Thus the second-order operator

$$
\mathcal{L} \stackrel{\text { def }}{=} \frac{1}{2} A_{1}^{2}+\frac{1}{2} A_{2}^{2}=\frac{1}{2} \frac{\partial^{2}}{\partial x^{2}}+\frac{x^{2}}{2} \frac{\partial^{2}}{\partial y^{2}}
$$

is hypoelliptic (in the literature, this operator is often attributed to Grushin). Now let $(\Omega, \mathcal{F}, \mathbb{P})$ be a probability triple on which is defined a Wiener process $W$. We are interested in the stochastic partial differential equations (SPDE's)

$$
\begin{aligned}
d p^{i} & =\mathcal{L} p^{i} d t+A_{i} p^{i} \circ d W_{t}, \quad t \geq 0, \\
p^{i}(0, \cdot) & =\delta_{(0,0)},
\end{aligned}
$$

where $\circ$ denotes Stratonovich integration. Specifically, we are interested in the behavior of these three SPDE's as $t$ tends to zero.

Received by the editors March 7, 1999 and, in revised form, December 6, 1999.

1991 Mathematics Subject Classification. Primary 60H15.

Key words and phrases. Fundamental solution, hypoellipticity, stochastic PDE's.

The author would like to thank the anonymous referee for a very careful reading of the manuscript. The author received support from NSF DMS-9726739 and NSF DMS-9615877 during the preparation of this work.

(C)2000 American Mathematical Society 
The background of our interest is the general study of SPDE's of the form

$$
\begin{aligned}
d u & =\mathcal{A} u d t+\mathcal{M} u \circ d W_{t}, \\
u(0, \cdot) & =\delta_{y}
\end{aligned}
$$

on $d$-dimensional differentiable manifolds, where $\mathcal{A}$ is a second-order partial-differential operator and $\mathcal{M}$ is a first-order partial-differential operator, and $y$ is some fixed point in $M$. Of course if $\mathcal{M} \equiv 0$ and $\mathcal{A}$ is elliptic, the results are classical; for "nice" $x$,

$$
u(t, x)=(2 \pi t)^{-d / 2} \exp \left[-\frac{d^{2}(x, y)}{2 t}+\mathfrak{W}_{y}(t, x)\right]\left\{a_{0}(x)+a_{1}(x) t+a_{2}(x) t^{2} \ldots\right\}
$$

as $t$ tends to zero, where $d$ is the Riemannian distance, $\mathfrak{W}_{y}(t, x)$ is the work done by the non-self-adjoint part of $\mathcal{A}$ along the geodesic from $y$ to $x$ (see [10]), $a_{0}$ is related to the Ruse Invariant (see [3]), and the $a_{i}$ 's are functions which can be iteratively derived according to calculations of Minakshisundaram and Pleijel (see [2] and also [4]). If $\mathcal{M} \equiv 0$ and $\mathcal{A}$ is hypoelliptic, Ben Arous in [1] and Leandre in [6] and [7] showed that a similar expansion holds if we interpret $d$ as the Carnot-Carathéodory distance (see also [8]). Since SPDE's where $\mathcal{M}$ is zero-order play a central role in filtering theory, it is natural to ask for similar expansions in the stochastic case. The first result was by Zhang [13, where the case of $\mathcal{A}$ elliptic and $\mathcal{M}$ zero-order was studied; one should replace the deterministic $a_{i}$ 's by a collection of iterated stochastic integrals. The case of $\mathcal{A}$ hypoelliptic and $\mathcal{M}$ zero-order was considered by Mesnager $[9]$.

It turns out that if $\mathcal{M}$ is first-order and $\mathcal{A}$ is elliptic, a new phenomenon occurs. The simplest incarnation of this occurs if the manifold is $\mathbb{R}, \mathcal{A}=\frac{1}{2} \frac{d^{2}}{d x^{2}}$, and $\mathcal{M}=\frac{d}{d x}$; then it is easily seen that the solution of (2) is

$$
\begin{aligned}
u(t, x) & =\exp \left[-\frac{\left|x-y+W_{t}\right|^{2}}{2 t}\right] / \sqrt{2 \pi t} \\
& =\exp \left[-\frac{|x-y|^{2}}{2 t}-\left(\frac{x-y}{t}\right) W_{t}-\frac{W_{t}^{2}}{2 t}\right] / \sqrt{2 \pi t}, \quad t \geq 0, x \in \mathbb{R} .
\end{aligned}
$$

Here the dominant exponential term is the standard distance function, the second term is the work done by od $W_{t}$ along the line joining $x$ and $y$ (i.e., the geodesic), and the third exponential term, which cannot be neglected, grows like $\log \log t^{-1}$ (due to the law of the iterated logarithm) but its statistics are bounded (i.e., it has a chi-square distribution). In general, the third term involves a randomly-forced Jacobi equation (see [11] and [12]). This brings us to the present work, which is a case study in the final situation, where $\mathcal{A}$ is hypoelliptic and $\mathcal{M}$ is first-order. We have chosen the simplest possible form of this problem to indicate what phenomena are at work. The operator $\mathcal{A}$ is of the Grushin type. This operator is degenerate only along the line $\{0\} \times \mathbb{R}$, so we choose our initial Dirac mass to be at $(0,0)$. By analogy with [12, we expect to see interesting phenomena when $\mathcal{M}$ has a first-order part; i.e., when $\mathcal{M}$ is a vector field. Since a general vector field can be written as a linear combination of $A_{1}, A_{2}$, and $A_{3}$, we consider these three cases separately. By understanding the behavior of this simple case, we should be able to glimpse a general theory for (2); we will develop this elsewhere.

The basic idea of our proof is to start along the road of [12. We shall rescale and then write a variational problem similar to one for a subRiemannian-type distance function. This variational problem is that of finding the control of least cost which 
drives a certain stochastic differential equation ( $\mathrm{SDE})$ from $(0,0)$ to $(x, y)$. We then must be careful with the variational problem. In essence, we must keep track of the directions in which the noise can drive the SDE versus the directions in which controls can drive the SDE, and the rates of each in terms of iterated Lie brackets. Finally, we note that our geometry is not locally constant since the dimension of the span of $A_{1}$ and $A_{2}$ is not constant (i.e., it is 1 along $\{0\} \times \mathbb{R}$ and 2 elsewhere).

\section{A RESCALING HEURISTIC}

We will first rescale the SPDE's (1); set

$$
q^{i, \varepsilon}(t, x, y) \stackrel{\text { def }}{=} p^{i}(t \varepsilon, x, y), \quad t \geq 0,(x, y) \in \mathbb{R}^{2},
$$

for all $\varepsilon>0$; thus

$$
p^{i}(t, x, y)=q^{i, t}(1, x, y)
$$

We note that $q^{i, \varepsilon}$ satisfies the SPDE

$$
\begin{aligned}
d q^{i, \varepsilon} & =\varepsilon \mathcal{L} q^{i, \varepsilon} d t+\varepsilon^{1 / 2} A_{i} q^{i, \varepsilon} \circ d W_{t}^{\varepsilon}, \quad t \geq 0, x \in \mathbb{R}^{2}, \\
q^{i, \varepsilon}(0, \cdot) & =\delta_{(0,0)},
\end{aligned}
$$

where

$$
W_{t}^{\varepsilon} \stackrel{\text { def }}{=} \varepsilon^{-1 / 2} W_{t \varepsilon}, \quad t \geq 0 .
$$

Next we will replace $W^{\varepsilon}$ by a smooth function $b$; this leads to the PDE

$$
\begin{aligned}
d q^{i, \varepsilon, b} & =\varepsilon \mathcal{L} q^{i, \varepsilon, b} d t+\varepsilon^{1 / 2} A_{i} q^{i, \varepsilon, b} \dot{b}(t) d t, \quad t \geq 0 . \\
q^{i, \varepsilon, b}(0, \cdot) & =\delta_{(0,0)},
\end{aligned}
$$

A reasonable guess is thus that

$$
q^{i, \varepsilon, b}(t, x, y) \asymp \exp \left[-\varepsilon^{-1} \tilde{J}_{i}^{\varepsilon, b}(t, x, y)\right]
$$

where

$$
\begin{array}{r}
\tilde{J}_{i}^{\varepsilon, b}(t, x, y) \stackrel{\text { def }}{=} \inf \left\{\frac{1}{2} \int_{0}^{t} \sum_{j \in\{1,2\}} p_{j}^{2}(s) d s:\right. \\
\dot{\zeta}(s)=\sum_{j \in\{1,2\}} A_{j}(\zeta(s)) p_{j}(s)+\varepsilon^{1 / 2} A_{i}(\zeta(s)) \dot{b}(s), \\
\zeta(0)=(0,0), \zeta(t)=(x, y)\} .
\end{array}
$$

We should now retrace our steps and get a formula for $\tilde{J}_{i}^{t, W^{t}}(1, x, y)$.

The main idea of [12] is that, in the elliptic case, one can differentiate the variational formula (3) with respect to $\varepsilon$. This gives the correct exponential expansion in that case. Things are not so simple here, however. In the simplest case, when $i=3$, we see that the $b$ term (which, being a substitute for the Wiener process $W^{\varepsilon}$, should be thought of as a generalized function) can drive $\zeta$ in the $A_{3}$ direction; if $y=0$, then the controls $p_{1}$ and $p_{2}$ need to compensate for this through the bracket of their respective vector fields, incurring a much greater cost than if $y \neq 0$. On the other hand, in the case $i=1, y=0$, the control $p$ may be directly used, which isn't so costly. 
To sort these things out, let's define a semiflow $\left\{\tilde{\psi}_{t}^{i, b, \varepsilon} ; t \geq 0\right\}$ of diffeomorphisms of $\mathbb{R}^{2}$ by

$$
\begin{aligned}
& \dot{\tilde{\psi}}_{s}^{i, b, \varepsilon}(x, y)=\varepsilon^{1 / 2} A_{i}\left(\tilde{\psi}_{s}^{i, b, \varepsilon}(x, y)\right) \dot{b}(s), \quad t \geq 0,(x, y) \in \mathbb{R}^{2} . \\
& \tilde{\psi}_{0}^{i, b, \varepsilon}(x, y)=(x, y),
\end{aligned}
$$

Recall that if $Z$ is a vector field on $\mathbb{R}^{2}$ and $\psi$ is a diffeomorphism from $\mathbb{R}^{2}$ to itself, the pullback of $Z$ through $\psi$ is the vector field $\psi_{*} Z$ on $\mathbb{R}^{2}$ defined by

$$
\left(\psi_{*} Z\right)(x, y) \stackrel{\text { def }}{=} D \psi Z\left(\psi^{-1}(x, y)\right), \quad(x, y) \in \mathbb{R}^{2} .
$$

We can then rewrite (3) by making the transformation $\zeta(s)=\tilde{\psi}_{s}^{i, b, \varepsilon}(\xi(s))$; we get that

$$
\begin{aligned}
& \tilde{J}_{i}^{\varepsilon, b}(t, x, y) \stackrel{\text { def }}{=} \inf \left\{\frac{1}{2} \int_{0}^{t} \sum_{j \in\{1,2\}} p_{j}^{2}(s) d s:\right. \\
& \dot{\xi}(s)=\sum_{j \in\{1,2\}}\left(\tilde{\psi}_{s}^{i, \varepsilon, b,-1}\right)_{*} A_{j}(\xi(s)) p_{j}(s) \\
& \left.\quad \xi(0)=(0,0), \tilde{\psi}_{t}^{i, \varepsilon, b}(\xi(t))=(x, y)\right\} .
\end{aligned}
$$

Note that with this representation, we can rigorously retrace our steps and get a quantity which should contain the dominant asymptotics of $p^{i}$. Let's first replace $\varepsilon$ by $t$ and $b$ by $W^{t}$. We define a stochastic semiflow $\left\{\psi_{t}^{i} ; t \geq 0\right\}$ of diffeomorphisms of $\mathbb{R}^{2}$ by

$$
\begin{aligned}
d \psi_{t}^{i}(x, y) & =A_{i}\left(\psi_{t}^{i}(x, y)\right) \circ d W_{t}, \quad t \geq 0,(x, y) \in \mathbb{R}^{2} . \\
\psi_{0}^{i}(x, y) & =(x, y),
\end{aligned}
$$

Note that if $b$ is close in some sense to $W^{t}$, then $\tilde{\psi}_{s}^{i, t, b}$ is close to $\psi_{s t}^{i}$ (this can be made precise, but we will not need to do so). Then we define

$$
\begin{aligned}
J_{i}(t, x, y) \stackrel{\text { def }}{=} \inf \left\{\frac{1}{2} \int_{0}^{1} \sum_{j \in\{1,2\}} p_{j}^{2}(s) d s:\right. \\
\dot{\xi}(s)=\sum_{j \in\{1,2\}}\left(\psi_{s t}^{i,-1}\right)_{*} A_{j}(\xi(s)) p_{j}(s), \\
\left.\xi(0)=(0,0), \psi_{t}^{i}(\xi(1))=(x, y)\right\} \\
=t \inf \left\{\frac{1}{2} \int_{0}^{t} \sum_{j \in\{1,2\}} p_{j}^{2}(s):\right. \\
\dot{\xi}(s)=\sum_{j \in\{1,2\}}\left(\psi_{s}^{i,-1}\right)_{*} A_{j}(\xi(s)) p_{j}(s), \\
\left.\xi(0)=(0,0), \psi_{t}^{i}(\xi(t))=(x, y)\right\} .
\end{aligned}
$$


We can now claim

Proposition 2.1. We have that $\mathbb{P}$-a.s.,

$$
\lim _{t \rightarrow 0} t \ln p^{i}(t, x, y)=-\lim _{t \rightarrow 0} J_{i}(t, x, y) .
$$

Proof. The proof is essentially that of [6] and [7]. We can write $p^{i}(t, x, y)$ as a weighted density (with respect to Lebesgue measure on $\mathbb{R}^{2}$ ) of an SDE with random coefficients (using standard techniques of decompositions of stochastic flows; see, for example, [5, Chapter 4]). Then [6] and [7] can be used.

Our goal is to simplify the right-hand side of (5). Not surprisingly, we should look at the subRiemannian distance between $(x, y)$ and the origin of $\mathbb{R}^{2}$; define

$$
\begin{aligned}
d_{0,0}^{2}(x, y)=t \inf \left\{\int_{0}^{t} \sum_{j \in\{1,2\}} \dot{\gamma}_{j}^{2}(s) d s: \gamma_{1}, \gamma_{2}\right. & \in C_{0}^{1}([0, t] ; \mathbb{R}), \\
& \left.\gamma_{1}(t)=x, \int_{0}^{t} \gamma_{1}(s) \dot{\gamma}_{2}(s) d s=y\right\}
\end{aligned}
$$

for all $(x, y) \in \mathbb{R}^{2}$, where $C_{0}^{1}([0, t] ; \mathbb{R})$ is the collection of differentiable functions $\gamma$ such that $\gamma(0)=0$ (we note that this definition does not in fact depend upon $t$ ). We note that for all $(x, y)$, there are $\gamma_{1}$ and $\gamma_{2}$ which achieve the minimum. Our main result is

Theorem 2.2. In all cases (i.e., for all $i \in\{1,2,3\}$ ), and for all $(x, y) \in \mathbb{R}^{2}$,

$$
\lim _{t \rightarrow 0} t \ln p^{i}(t, x, y)=-\frac{d_{0,0}^{2}(x, y)}{2}, \quad \mathbb{P} \text {-a.s. }
$$

Proof. Lemmas 3.1, 4.3, and 5.3.

The motivation of our work is that the dependence of $J_{i}(t, x, y)$ of (4) upon $W$ is fairly explicit, so the proof of Theorem 2.2 should involve a minimum of technicalities and should thus allow us to focus on relevant qualitative phenomena. First, note that $\left\{\psi_{t}^{i,-1} ; t \geq 0\right\}$ describes a stochastic flow of diffeomorphisms of $\mathbb{R}^{2}$ given by

$$
\begin{aligned}
d \psi_{s}^{i,-1}(x, y) & =-\left(\psi_{t}^{i,-1}\right)_{*} A_{i}\left(\psi_{s}^{i,-1}(x, y)\right) \circ d W_{t}, \quad t \geq 0,(x, y) \in \mathbb{R}^{2} . \\
\psi_{0}^{i,-1}(x, y) & =(x, y),
\end{aligned}
$$

Second, note that for any $(x, y) \in \mathbb{R}^{2}$ and any vector field $V$ on $\mathbb{R}^{2},\left\{\left(\psi_{s}^{i,-1}\right)_{*} V(x, y)\right.$; $t \geq 0\}$ satisfies the $T_{(x, y)} \mathbb{R}^{2}$-valued stochastic differential equation

$$
\begin{aligned}
d\left(\left(\psi_{t}^{i,-1}\right)_{*} V\right)(x, y) & =\left(\left(\psi_{t}^{i,-1}\right)_{*}\left[A_{i}, V\right]\right)(x, y) \circ d W_{t}, \quad t \geq 0,(x, y) \in \mathbb{R}^{2} . \\
\left(\psi_{0}^{i,-1} V\right)(x, y) & =V(x, y),
\end{aligned}
$$

In the next three sections we will use this last fact to write a stochastic Taylor series for the terms in the minimization problem in (5); this will give us explicit dependencies on $W$. Since $A_{1}, A_{2}$, and $A_{3}$ are linear, the $\psi_{t}^{i}$ 's can be explicitly solved for. Along the same lines, it can be seen that $\left[A_{1}, A_{3}\right]=\left[A_{2}, A_{3}\right]=0$, so $\left(\psi_{t}^{i,-1}\right)_{*} A_{1}$ and $\left(\psi_{t}^{i,-1}\right)_{*} A_{2}$ can be explicitly represented. 
Before starting our case-by-case analysis, let's make some definitions. For $t>0$ and $\kappa \in(0,1 / 2)$, define

$$
[W]_{t, \kappa} \stackrel{\text { def }}{=} \sup _{0<s \leq t} \frac{\left|W_{s}\right|}{s^{\kappa}} .
$$

Lemma 2.3. For any $t>0, \kappa \in(0,1 / 2)$, and $\zeta \in L^{2}([0, t])$,

$$
\left|\int_{0}^{t} W_{s} \zeta(s) d s\right| \leq[W]_{t, \kappa} t^{\kappa+1 / 2}\left\{\int_{0}^{t} \zeta^{2}(s) d s\right\}^{1 / 2} .
$$

Proof. A simple application of Hölder's inequality.

Also note that for any $\alpha \in \mathbb{R}$ and $\varepsilon>0$,

$$
(1+\alpha)^{2} \leq\left(1+\varepsilon^{2}\right)+\left(1+\varepsilon^{-2}\right) \alpha^{2},
$$

as one can see from Young's inequality. Thirdly for each $\varepsilon \in \mathbb{R}$ and $y \in \mathbb{R}$, we define

$$
y_{\varepsilon} \stackrel{\text { def }}{=} \begin{cases}y & \text { if } y \neq 0 \\ \varepsilon & \text { if } y=0\end{cases}
$$

\section{CASE 3}

First, we consider the case $i=3$. This is the easiest case. We have

$$
\psi_{t}^{i}(x, y)=\left(x, y+W_{t}\right), \quad t \geq 0,(x, y) \in \mathbb{R}^{2},
$$

and due to (6) and (7), or direct calculations, we know that

$$
\left(\psi_{t}^{i,-1}\right)_{*} A_{1}=A_{1} \quad \text { and } \quad\left(\psi_{t}^{i,-1}\right)_{*} A_{2}=A_{2}
$$

Thus the minimization problem is

$$
\begin{gathered}
J_{3}(t, x, y)=t \inf \left\{\frac{1}{2} \int_{0}^{t} \sum_{j \in\{1,2\}} p_{j}^{2}(s) d s:\right. \\
\dot{\xi}(s)=\sum_{j \in\{1,2\}} A_{j}(\xi(s)) p_{j}(s), \\
\left.\xi(0)=(0,0), \xi(t)=\left(x, y-W_{t}\right)\right\} \\
=\frac{d_{0,0}^{2}\left(x, y-W_{t}\right)}{2} .
\end{gathered}
$$

Thus we have

Proposition 3.1. For any $(x, y) \in \mathbb{R}^{2}$,

$$
\lim _{t \rightarrow 0} t \ln p^{3}(t, x, y)=-\frac{d_{0,0}^{2}(x, y)}{2}, \quad \mathbb{P} \text {-a.s. }
$$




\section{CASE 2}

Next we consider the case $i=2$. This is the next-to-easiest case. We explicitly have that

$$
\psi_{t}^{i}(x, y)=\left(x, y+x W_{t}\right), \quad t \geq 0,(x, y) \in \mathbb{R}^{2},
$$

and due to (6) and (7), or direct calculations, we know that

$$
\left(\psi_{t}^{i,-1}\right)_{*} A_{1}=A_{1}-A_{3} W_{t} \quad \text { and } \quad\left(\psi_{t}^{i,-1}\right)_{*} A_{2}=A_{2} .
$$

Thus we can rewrite the minimization problem (4) as

$$
\begin{aligned}
J_{2}(t, x, y)=t \inf \left\{\frac{1}{2} \int_{0}^{t} \dot{\gamma}_{1}^{2}(s)+\dot{\gamma}_{2}^{2}(s) d s: \gamma_{1}, \gamma_{2} \in C_{0}^{1}([0, t] ; \mathbb{R})\right. \\
\left.\gamma_{1}(t)=x, \int_{0}^{t} \gamma_{1}(s) \dot{\gamma}_{2}(s) d s=y+\int_{0}^{t}\left\{W_{s}-W_{t}\right\} \dot{\gamma}_{1}(s) d s\right\} .
\end{aligned}
$$

An upper bound is

Lemma 4.1. We have that

$$
J_{2}(t, x, y) \leq \frac{d_{0,0}^{2}\left(x, y_{\varepsilon}\right)}{2}\left\{\left(1+\varepsilon^{2}\right)+4[W]_{t, \kappa}^{2} y_{\varepsilon}^{-2} t^{2 \kappa} d_{0,0}^{2}\left(x, y_{\varepsilon}\right)\left(1+\varepsilon^{-2}\right)\right\} .
$$

Proof. Let $\gamma_{1}$ and $\gamma_{2}$ in $C_{0}^{1}([0, t] ; \mathbb{R})$ be such that $\gamma_{1}(t)=x, \int_{0}^{t} \gamma_{1}(s) \dot{\gamma}_{2}(s) d s=y_{\varepsilon}$, and

$$
\int_{0}^{t} \dot{\gamma}_{1}^{2}(s)+\dot{\gamma}_{2}^{2}(s)=\frac{d_{0,0}^{2}\left(x, y_{\varepsilon}\right)}{t} .
$$

Now set $\zeta_{1} \stackrel{\text { def }}{=} \alpha_{\varepsilon} \gamma_{1}$ and $\zeta_{2}(s) \stackrel{\text { def }}{=} \beta_{\varepsilon} \gamma_{2}$. We want to choose $\alpha_{\varepsilon}$ and $\beta_{\varepsilon}$ such that $\zeta_{1}$ and $\zeta_{2}$ are an admissible pair for the variational problem for $J_{2}(t, x, y)$. Thus we need $\alpha_{\varepsilon}=1$. We also need that

$$
\beta_{\varepsilon} y_{\varepsilon}=y+\int_{0}^{t} \dot{\gamma}_{1}(s)\left\{W_{s}-W_{t}\right\} d s .
$$

Thus there are two possibilities. If $y \neq 0$, then we need that

$$
\beta_{\varepsilon}=1+y^{-1} \int_{0}^{t} \dot{\gamma}_{1}(s)\left\{W_{s}-W_{t}\right\} d s
$$

and if $y=0$, then we need that

$$
\beta_{\varepsilon}=\varepsilon^{-1} \int_{0}^{t} \dot{\gamma}_{1}(s)\left\{W_{s}-W_{t}\right\} d s .
$$

Thus

$$
\beta_{\varepsilon}=\chi_{\{y \neq 0\}}+y_{\varepsilon}^{-1} \int_{0}^{t} \dot{\gamma}_{1}(s)\left\{W_{s}-W_{t}\right\} d s .
$$

From Lemma 2.3, we get that

$$
\left|\int_{0}^{t} \dot{\gamma}_{1}(s)\left\{W_{s}-W_{t}\right\} d s\right| \leq 2[W]_{t, \kappa} t^{\kappa} d_{0,0}\left(x, y_{\varepsilon}\right) .
$$

Thus

$$
\beta_{\varepsilon}^{2} \leq\left(1+\varepsilon^{2}\right)+4[W]_{t, \kappa}^{2} y_{\varepsilon}^{-2} t^{2 \kappa} d_{0,0}^{2}\left(x, y_{\varepsilon}\right)\left(1+\varepsilon^{-2}\right) .
$$

This gives us (9). 
From this we get a lower bound.

Lemma 4.2. We have that

$$
J_{2}(t, x, y) \geq \frac{\inf _{\left|y^{\prime}-y\right| \leq 4[W]_{t, \kappa} t^{\kappa+1 / 2} J_{2}^{1 / 2}(t, x, y)} d_{0,0}^{2}\left(x, y^{\prime}\right)}{2} .
$$

Proof. If $\gamma_{1}$ and $\gamma_{2}$ are sufficiently close to being a minimizer of (8), we must have that

$$
\begin{aligned}
\left|\int_{0}^{t} \dot{\gamma}_{1}(s)\left\{W_{s}-W_{t}\right\} d s\right| \leq 2[W]_{t, \kappa} t^{\kappa+1 / 2}\left\{\int_{0}^{t} \dot{\gamma}_{1}^{2}(s) d s\right\}^{1 / 2} \\
\leq 2^{3 / 2}[W]_{t, \kappa} t^{\kappa+1 / 2}\left\{\frac{1}{2} \int_{0}^{t} \dot{\gamma}_{1}^{2}(s) d s\right\}^{1 / 2} \leq 4[W]_{t, \kappa} t^{\kappa+1 / 2} J_{2}^{1 / 2}(t, x, y) .
\end{aligned}
$$

Thus

$$
\begin{aligned}
J_{2}(t, x, y) \geq t \inf \left\{\frac{1}{2} \int_{0}^{t} \dot{\gamma}_{1}^{2}(s)+\dot{\gamma}_{2}^{2}(s) d s: \gamma_{1}, \gamma_{2} \in C_{0}^{1}([0, t] ; \mathbb{R})\right. \\
\left.\gamma_{1}(t)=x,\left|\int_{0}^{t} \gamma_{1}(s) \dot{\gamma}_{2}(s) d s-y\right| \leq 4[W]_{t, \kappa} t^{\kappa+1 / 2} J_{2}^{1 / 2}(t, x, y)\right\} .
\end{aligned}
$$

This gives the result.

The combination of these gives

Proposition 4.3. For any $(x, y) \in \mathbb{R}^{2}$,

$$
\lim _{t \rightarrow 0} t \ln p^{2}(t, x, y)=-\frac{d_{0,0}^{2}(x, y)}{2}, \quad \mathbb{P} \text {-a.s. }
$$

Proof. First take the limit in $t$, then in $\varepsilon$.

\section{CASE 1}

We finally consider the case $i=1$. We explicitly have that

$$
\phi_{t}^{i}(x, y)=\left(x+W_{t}, y\right), \quad t \geq 0,(x, y) \in \mathbb{R}^{2},
$$

and due to (6) and (7), or direct calculations, we know that

$$
\left(\psi_{t}^{i,-1}\right)_{*} A_{1}=A_{1} \quad \text { and } \quad\left(\psi_{t}^{i,-1}\right)_{*} A_{2}=A_{2}+A_{3} W_{t} .
$$

Thus the minimization problem is

$$
\begin{array}{r}
J_{1}(t, x, y)=t \inf \left\{\frac{1}{2} \int_{0}^{t} \dot{\gamma}_{1}^{2}(s)+\dot{\gamma}_{2}^{2}(s) d s: \gamma_{1}, \gamma_{2} \in C_{0}^{1}\left([0, t] ; \mathbb{R}^{2}\right)\right. \\
\left.\gamma_{1}(t)=x-W_{t}, \int_{0}^{t}\left\{\gamma_{1}(s)+W_{s}\right\} \dot{\gamma}_{2}(s) d s=y\right\} .
\end{array}
$$

The upper bound is

Lemma 5.1. We have that

$$
J_{1}(t, x, y) \leq \frac{d_{0,0}^{2}\left(x-W_{t}, y_{\varepsilon}\right)}{2}\left\{\left(1+\varepsilon^{2}\right)+2[W]_{t, \kappa}^{2} y_{\varepsilon}^{-2} t^{2 \kappa} d_{0,0}^{2}\left(x-W_{t}, y_{\varepsilon}\right)\left(1+\varepsilon^{-2}\right)\right\} .
$$


Proof. Let $\gamma_{1}$ and $\gamma_{2}$ in $C_{0}^{1}([0, t] ; \mathbb{R})$ be such that $\gamma_{1}(t)=x-W_{t}, \int_{0}^{t} \gamma_{1}(s) \dot{\gamma}_{2}(s) d s=$ $y_{\varepsilon}$, and

$$
\int_{0}^{t} \dot{\gamma}_{1}^{2}(s)+\dot{\gamma}_{2}^{2}(s)=\frac{d_{0,0}^{2}\left(x-W_{t}, y_{\varepsilon}\right)}{t} .
$$

Now set $\zeta_{1} \stackrel{\text { def }}{=} \alpha_{\varepsilon} \gamma_{1}$ and $\zeta_{2}(s) \stackrel{\text { def }}{=} \beta_{\varepsilon} \gamma_{2}$. We want to choose $\alpha_{\varepsilon}$ and $\beta_{\varepsilon}$ such that $\zeta_{1}$ and $\zeta_{2}$ are an admissible pair for the variational problem for $J_{1}(t, x, y)$. Thus we need $\alpha_{\varepsilon}=1$. We also need that

$$
\beta_{\varepsilon} y_{\varepsilon}=y+\int_{0}^{t} \dot{\gamma}_{1}(s)\left\{W_{s}-W_{t}\right\} d s .
$$

Thus there are two possibilities. If $y \neq 0$, then we need that

$$
\beta_{\varepsilon}=1-y^{-1} \int_{0}^{t} \dot{\gamma}_{2}(s)\left\{W_{s}-W_{t}\right\} d s
$$

and if $y=0$, then we need that

$$
\beta_{\varepsilon}=-\varepsilon^{-1} \int_{0}^{t} \dot{\gamma}_{2}(s)\left\{W_{s}-W_{t}\right\} d s .
$$

Thus

$$
\beta_{\varepsilon}=\chi_{\{y \neq 0\}}-y_{\varepsilon}^{-1} \int_{0}^{t} \dot{\gamma}_{2}(s) W_{s} d s .
$$

From Lemma 2.3, we get that

$$
\left|\int_{0}^{t} \dot{\gamma}_{2}(s) W_{s} d s\right| \leq[W]_{t, \kappa} t^{2 \kappa} d_{0,0}^{2}\left(x-W_{t}, y_{\varepsilon}\right) .
$$

Thus

$$
\beta_{\varepsilon}^{2} \leq\left(1+\varepsilon^{2}\right)+[W]_{t, \kappa}^{2} y_{\varepsilon}^{-2} t^{2 \kappa} d_{0,0}^{2}\left(x-W_{t}, y_{\varepsilon}\right)\left(1+\varepsilon^{-2}\right) .
$$

This gives us (11).

From this we get a lower bound.

Lemma 5.2. We have that

$$
J_{1}(t, x, y) \geq \frac{\inf _{\left|y^{\prime}-y\right| \leq 2^{3 / 2}[W]_{t, \kappa} t^{\kappa+1 / 2} J_{1}^{1 / 2}(t, x, y)} d_{0,0}^{2}\left(x-W_{t}, y^{\prime}\right)}{2} .
$$

Proof. If $\gamma_{1}$ and $\gamma_{2}$ are sufficiently close to being a minimizer of (10), we must have that

$$
\left|\int_{0}^{t} \dot{\gamma}_{2}(s) W_{s} d s\right| \leq 2^{1 / 2}[W]_{t, \kappa} J_{1}^{1 / 2}(t, x, y)
$$

Thus

$$
\begin{aligned}
J_{1}(t, x, y) \geq t \inf \left\{\frac{1}{2} \int_{0}^{t} \dot{\gamma}_{1}^{2}(s)+\dot{\gamma}_{2}^{2}(s) d s: \gamma_{1}, \gamma_{2} \in C_{0}^{1}([0, t] ; \mathbb{R})\right. \\
\left.\gamma_{1}(t)=x,\left|\int_{0}^{t} \gamma_{1}(s) \dot{\gamma}_{2}(s) d s-y\right| \leq 2^{1 / 2}[W]_{t, \kappa} t^{\kappa+1 / 2} J_{1}^{1 / 2}(t, x, y)\right\} .
\end{aligned}
$$

This gives the result. 
The combination of these gives

Proposition 5.3. For any $(x, y) \in \mathbb{R}^{2}$,

$$
\lim _{t \rightarrow 0} t \ln p_{1}(t, x, y)=-\frac{d_{0,0}^{2}(x, y)}{2}, \quad \mathbb{P} \text {-a.s. }
$$

Proof. First take the limit in $t$, then in $\varepsilon$.

\section{REFERENCES}

1. G. Ben Arous, Développement asymptotique du noyau de la chaleur hypoelliptique hors du cut-locus, Ann. Sci. École Norm. Sup. (4) 21 (1988), 307-331. MR 89k:60087

2. I. Chavel, Eigenvalues in Riemannian geometry, Pure and Applied Mathematics, 115, Academic Press, 1984. MR 86g:58140

3. K. D. Elworthy, Stochastic differential equations on manifolds, London Mathematical Society Lecture Note Series, 70, Cambridge University Press, New York, 1982. MR 84d:58080

4. Y. Kannai, Off diagonal short time asymptotics for solutions of diffusion equations, Communications in P.D.E.'s 2 (1977), 781-830. MR 58:29247

5. H. Kunita, Stochastic Flows and Stochastic Differential Equations, Cambridge University Press, New York, 1990. MR 91m:60107

6. R. Léandre, Majoration en temps petit de la densité d'une diffusion dégénérée, Probability Theory and Related Fields 74 (1987), 289-294. MR 88c:60144

7. Minoration en temps petit de la densité d'une diffusion dégénérée, Journal of Functional Analysis 74 (1987), 399-414. MR 88k:60147

8. _ Développement asymptotique de la densité de diffusions dégénérées, Forum Math. 4 (1992), 45-75. MR 93d:60100.

9. L. Mesnager, Estimation en temps petit de densités conditionelles dan des problemes de filtrage nonlineare, Ph.D. Thesis, Université de Paris-Sud, 1996.

10. S. A. Molchanov, Diffusion processes, and Riemannian geometry, Uspehi Mat. Nauk 30 (1975), 3-59. MR 54:1404

11. R. B. Sowers, Recent results on the short-time geometry of random heat kernels, Math. Res. Lett. 1 (1994), 663-675. MR 95m:58133

12. Short-time geometry of random heat kernels, Mem. Amer. Math. Soc. 132 (1998). MR 98i:60060

13. H. Zhang, Développement en temps petit de la solution de l'équation de Zakai et résolution numérique par maillage adaptatif, Ph.D. Thesis, Université de Provence-Centre Saint-Charles, 1992.

Department of Mathematics, University of Illinois, 1409 W. Green Street, Urbana, ILLINOIS 60201

E-mail address: r-sowers@math.uiuc.edu 